\title{
Variant Achalasia: A New Category of the Chicago Classification Published in 2011
}

Hyung Hun Kim, Moo In Park, ${ }^{*}$ Jong Hyun Baik, Seun Ja Park and Won Moon

Department of Internal Medicine, Kosin University College of Medicine, Busan, Korea

A 56-year-old woman presented to our outpatient clinic because she had been having swallowing difficulty of solid food and liquid for the past month. Intense psychiatric stress and eating quickly provoked dysphagia, but exercise relieved the symptoms.
The physical examination was unremarkable, and there were no abnormal findings on esophagogastroduodenoscopy. High-resolution manometry revealed an elevated mean integrated relaxation pressure (IRP) of $15.5 \mathrm{mmHg}$. Additionally, a simultaneous
A

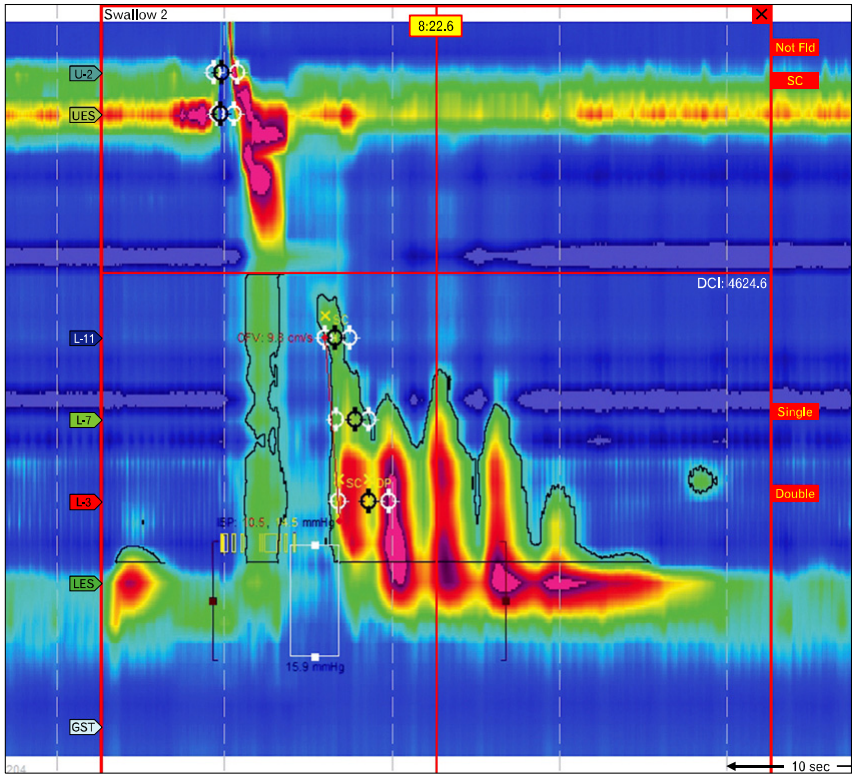

B

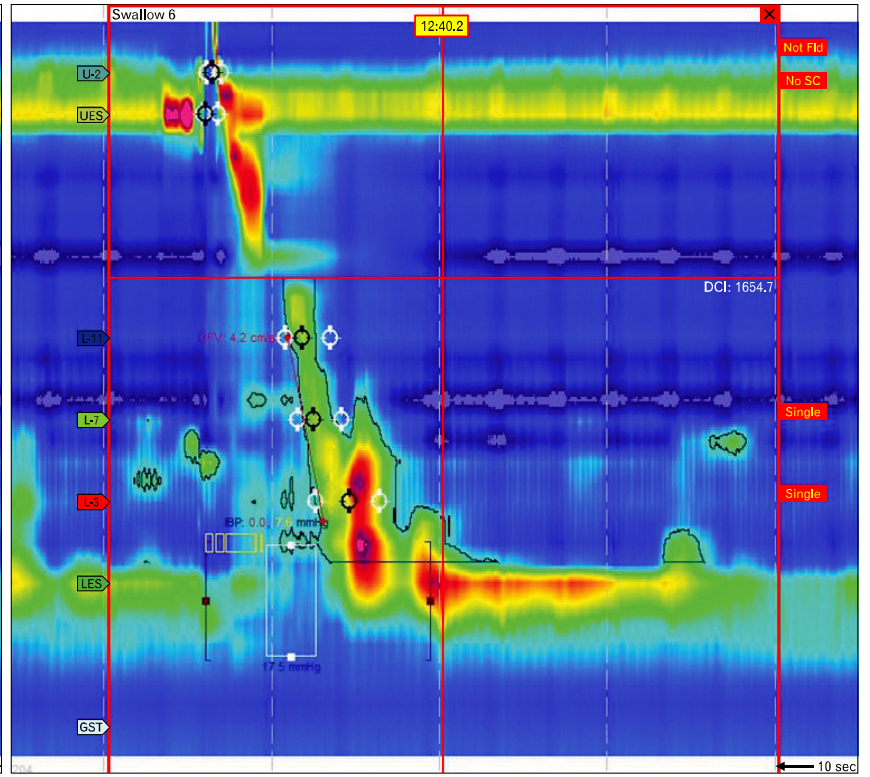

Figure 1. High-resolution manometry (HRM) spatio-temporal plots. (A) HRM revealed elevated integrated relaxation pressure (15.9 $\mathrm{mmHg})$ with increased contractile front velocity $(9.8 \mathrm{~cm} / \mathrm{sec})$. (B) $\mathrm{HRM}$ also showed increased integrated relaxation pressure $(17.5 \mathrm{mmHg})$ and normal peristaltic contraction and contractile front velocity $(4.2 \mathrm{~cm} / \mathrm{sec})$.

Received: October 28, 2011 Revised: October 30, 2011 Accepted: November 1, 2011

(c) This is an Open Access article distributed under the terms of the Creative Commons Attribution Non-Commercial License (http://creativecommons. org/licenses/by-nc/3.0) which permits unrestricted non-commercial use, distribution, and reproduction in any medium, provided the original work is properly cited.

*Correspondence: Moo In Park, MD

Department of Internal Medicine, Kosin University College of Medicine, 34 Amnam-dong, Seo-gu, Busan 602-702, Korea

Tel: +82-51-990-5205, Fax: +82-51-990-5055, E-mail: mipark@ns.kosinmed

Financial support: None.

Conflicts of interest: None. 


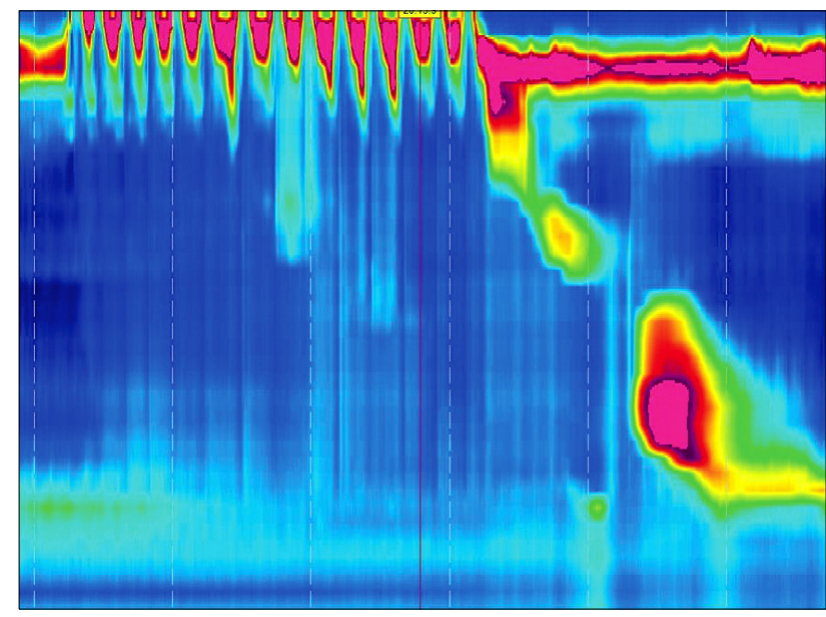

Figure 2. Normal peristalsis with deglutitive inhibition was observed on the multiple swallow test.

contraction was observed in 6 out of 10 swallows (Fig. 1). There were no abnormalities noted on a multiple swallow test (Fig. 2).

According to the Chicago classification published in 2008, it was impossible to define the combination of these 2 findings with a single term. ${ }^{1}$ Because of the elevated mean IRP, this presentation was initially classified as impaired esophagogastric junction (EGJ) relaxation. However, there was no category consistent with both the simultaneous contraction and elevated contractile front velocity in impaired EGJ relaxation. Aside from the elevated mean IRP, this presentation was compatible with distal esophageal spasm with a contractile front velocity $>8 \mathrm{~cm} / \mathrm{sec}$ in $20 \%$ of swallows. Since esophageal spasm occasionally accompanies impaired EGJ relaxation, we diagnosed the patient as distal esophageal spasm with impaired EGJ relaxation. ${ }^{2}$

However, the revised Chicago classification published in 2011 classifies this presentation as variant achalasia. ${ }^{3}$ This new classification enabled us to categorize our findings as EGJ outflow obstruction due to elevated IRP and some instances of intact peristalsis. Thereafter, we have been able to diagnose this presentation as variant achalasia more specifically after ruling out the mechanical obstruction and hypercontractility. ${ }^{3}$ Our case exhibited characteristic findings of the newly proposed category in the 2011 Chicago classification, which addresses the findings not classifiable by the 2008 Chicago classification system.

\section{References}

1. Kahrilas PJ, Ghosh SK, Pandolfino JE. Esophageal motility disorders in terms of pressure topography: the Chicago Classification. J Clin Gastroenterol 2008;42:627-635.

2. Almansa C, Heckman MG, Devault KR, Bouras E, Achem SR Esophageal spasm: demographic, clinical, radiographic, and manometric features in 108 patients. Dis Esophagus Published Online First: 23 Sep 2011. doi: 10.1111/j.1442-2050.2011.01258.x.

3. Bredenoord AJ, Fox M, Kahrilas PJ, et al. Chicago classification criteria of esophageal motility disorders defined in high resolution esophageal pressure topography (EPR). International High Resoultion Manometry Consensus Meeting 2011, April, Ascona, Switzerland:1-23. 